\title{
Mejorar la motivación en el aula mediante el uso de plataformas de aprendizaje basadas en juegos: Quizlet vs Kahoot
}

\author{
Consuelo Calafat Marzal ${ }^{a}$, Rosa Puertas Medina ${ }^{b}, M^{a} L u i s a$ Martí Selva ${ }^{c}$ \\ a,b,c Departamento de Economía y Ciencias Sociales, Universidad Politécnica de Valencia, Camino de \\ Vera s/n, Valencia. macamar3@esp.upv.es, rpuestas@esp.upv.es, mlmarti@esp.upv.es
}

\section{Resumen}

Las plataformas de juegos son cada vez más demandadas por los alumnos en cualquier nivel educativo. La utilización de estas herramientas para incrementar la motivación de los alumnos en grupos numerosos de asignaturas universitarias favorece una mayor asistencia y participación del alumno a las clases presenciales. Para los estudiantes es un estímulo interactivo y divertido que permite introducir o repasar contenidos. Mediante su utilización se potencia la motivación, la concentración y el esfuerzo de los alumnos, especialmente durante el desarrollo de las clases donde se fomenta el trabajo colaborativo y mejora la comunicación, tanto entre alumnos como con el profesor. Se utiliza como medio de juego sus teléfonos móviles o los ordenadores del aula. Los profesores tienen que seleccionar las unidades de estudio y preparar previamente los juegos adaptándose a los entornos de estas plataformas, posteriormente le permitirán evaluar la asimilación de los contenidos por parte de los alumnos, y por tanto tener un feedback a lo largo de curso.

El objetivo de este estudio es analizar las plataformas de juegos como herramienta para mejorar la motivación de los alumnos en el aula. Se compararán dos plataformas, como son Quizlet live y Kahoot. En las dos se permite la realización de juegos basados en preguntas tipo test. Además, en Quizlet live se permite la preparación de otras actividades, como son diagramas interactivos, juegos de relacionar contenidos, etc. Aunque en las dos plataformas muchos usuarios han dejado en abierto actividades preparadas, es más recomendable realizar las propias actividades para que se adapten adecuadamente tanto a la asignatura como al nivel exigido por el profesor.

Palabras clave: juego en red; educación; cuestionarios online; motivación; aprendizaje colaborativo 
Mejorar la motivación en el aula mediante el uso de plataformas de aprendizaje basadas en juegos: Quizlet vs Kahoot

\section{Introducción}

La innovación educativa, en el contexto universitario, trata de dar respuesta a múltiples retos que reclaman una renovación de los procesos de enseñanza-aprendizaje. Las prácticas educativas más tradicionales van cambiando por aquéllas en las que se reflejan los últimos avances en metodologías, entornos de aprendizaje y sistemas de evaluación más dinámicos (Gutiérrez et al., 2010). Las herramientas Web 2.0 permiten un cambio en el modelo de aprendizaje, de una web (1.0) con un modelo de aprendizaje lineal a una web (2.0) dinámica y participativa con un modelo de aprendizaje interactivo y colaborativo (Carreño y Velez, 2016). Además, según Rodríguez (2008) la web 2.0 está en constante cambio, como se manifiesta en el conjunto plataformas, aplicaciones y servicios que promueven la participación y creación, publicación y diseminación de contenidos. En la misma línea, Vivancos (2010) describe las Tecnologías del Aprendizaje y el Conocimiento (TAC) como el know-how ofrecido a la educación, refiriéndose a como las Tecnologías de la Información y la Comunicación (TIC) se ponen al servicio directo de la educación para el aprovechamiento del aprendizaje y el conocimiento. La utilización de estas herramientas beneficia tanto a profesores como a alumnos (Salgado et al., 2013, Tabuenca et al., 2013), y suponen una intensificación de la participación activa en dicho proceso de aprendizaje (Salinas, 2004).

Entre las nuevas técnicas de formación y motivación se encuentran las basadas en los juegos en el aula (game-based learning), utilizadas para realizar cuestionarios y entretenimientos online dentro del aula, favoreciendo la motivación mediante la competición entre los alumnos (Wang, 2015). Estas tareas se pueden plantear tanto para obtener una retroalimentación de los contenidos impartidos en un tema o una clase (Pintro et al., 2015) como para valorar los conocimientos del alumno antes de empezar determinados contenidos. Es decir, permiten obtener en tiempo real información sobre el progreso en el aprendizaje, para detectar los problemas de comprensión o las carencias de forma rápida (Fuentes et al., 2016).

Una de las principales ventajas de esta nueva forma de aprender es la participación activa de todos los alumnos. Los estudiantes más tímidos y callados, que trabajan bien pero pasan desapercibidos, se hacen presentes en la clase al alcanzar puestos destacados en el marcador. Esta metodología de aprendizaje permite acortar la "distancia" con los alumnos/as distantes, una característica de los enfoques de enseñanza centrados en el estudiante (Salinas, 2004). Según Kay y LeSage (2009) la implementación de estos sistemas de respuesta personal en el aula genera los siguientes efectos positivos:

a) Respecto del ambiente en el aula, el alumno aumenta su atención y participa activamente en la resolución de las cuestiones planteados, compite con otros alumnos/as, en la resolución de problemas. 
b) Respecto del aprendizaje en sí, se produce una mejora notable gracias a la interacción que estos sistemas provoca entre los alumnos/as de la clase, discutiendo sobre cuál es la solución adecuada y porqué.

c) Respecto de la evaluación, se posibilita la obtención regular de una retroalimentación, tanto para el profesor como para el estudiante de la calidad de su enseñanza y del nivel de su aprendizaje, respectivamente.

El objetivo de este estudio es analizar las plataformas de juegos como herramienta para mejorar la motivación de los alumnos en el aula. Se compararán dos plataformas, como son Kahoot! y Quizlet live. Ambas han sido utilizadas en la asignatura de Economía Política (EP) de primer curso del Grado de Gestión y Administración Pública (GGAP) de la Facultad de Administración y Dirección de Empresas (FADE) de la Universitat Politècnica de València (UPV). Esta asignatura tiene un total de 6 créditos ECTS y se imparte en el primer cuatrimestre de primer curso, y tiene un total de 52 alumnos en el grupo de la tarde. Los contenidos se dividen en dos bloques: microeconomía compuesto por 4 unidades didácticas y macroeconomía desarrollado también en 4 temas. En la parte de microeconomía se diseñaron juegos en el aula mediante Kahoot! y en macroeconomía mediante Quizlet. Éstos se plantearon al finalizar los temas en los que los alumnos habían mostrado mayor dificultad, y se utilizó unos 20 minutos para su realización. Los cuestionarios fueron planteados por los docentes de la asignatura con objeto de adaptarse lo más posible al nivel exigido, aunque en las dos plataformas muchos usuarios han dejado en abierto actividades preparadas.

El artículo se estructura de la siguiente manera. En la sección siguiente se realiza un descripción y procedimiento de uso de la plataforma kahoot|. En la sección tercera, se analiza la plataforma Quizlet Live. Por último, en la cuarta sección se muestran las principales conclusiones del estudio.

\section{Descripción de la muestra}

La asignatura de Economía Política (EP) de primer curso del Grado de Gestión y Administración Pública (GGAP) de la de la Facultad de Administración y Dirección de Empresas (FADE) de la Universitat Politècnica de València (UPV) tiene un total de 6 créditos ECTS y se imparte en el primer cuatrimestre de primer curso, y tiene un total de 52 alumnos en el grupo de la tarde. Los contenidos se dividen en dos bloques: microeconomía compuesto por 4 unidades didácticas y macroeconomía desarrollados también en 4 unidades.

El rendimiento de los alumnos alcanzado en la asignatura de EP ha mejorado en los últimos años pero sigue siento inferior al que alcanzan en el resto de asignaturas del mismo curso. 
Mejorar la motivación en el aula mediante el uso de plataformas de aprendizaje basadas en juegos: Quizlet vs Kahoot

Ello ha conducido en varias ocasiones que los docentes realizaran una reflexión sobre las posibles causas y soluciones viables (Puertas et al, 2015; Calafat et al., 2017).

La evolución del número de aprobados/suspensos/no presentados en EP durante los últimos cursos académicos no refleja un patrón de comportamiento consolidado que pueda considerarse mantenible en los próximos años. En 2012-2013 y 2016-2017 se logró una mejora significativa que, en opinión de los docentes, podría deberse a las innovaciones introducidas, sin embargo no se consolidó y se trató de algo puntual (Figura 1). Es por ello, que se están introduciendo nuevas metodologías en la asignatura con el objetivo de mejorar la motivación en las clases $y$, como consecuencia, una mejora en el rendimiento de los alumnos en la asignatura.

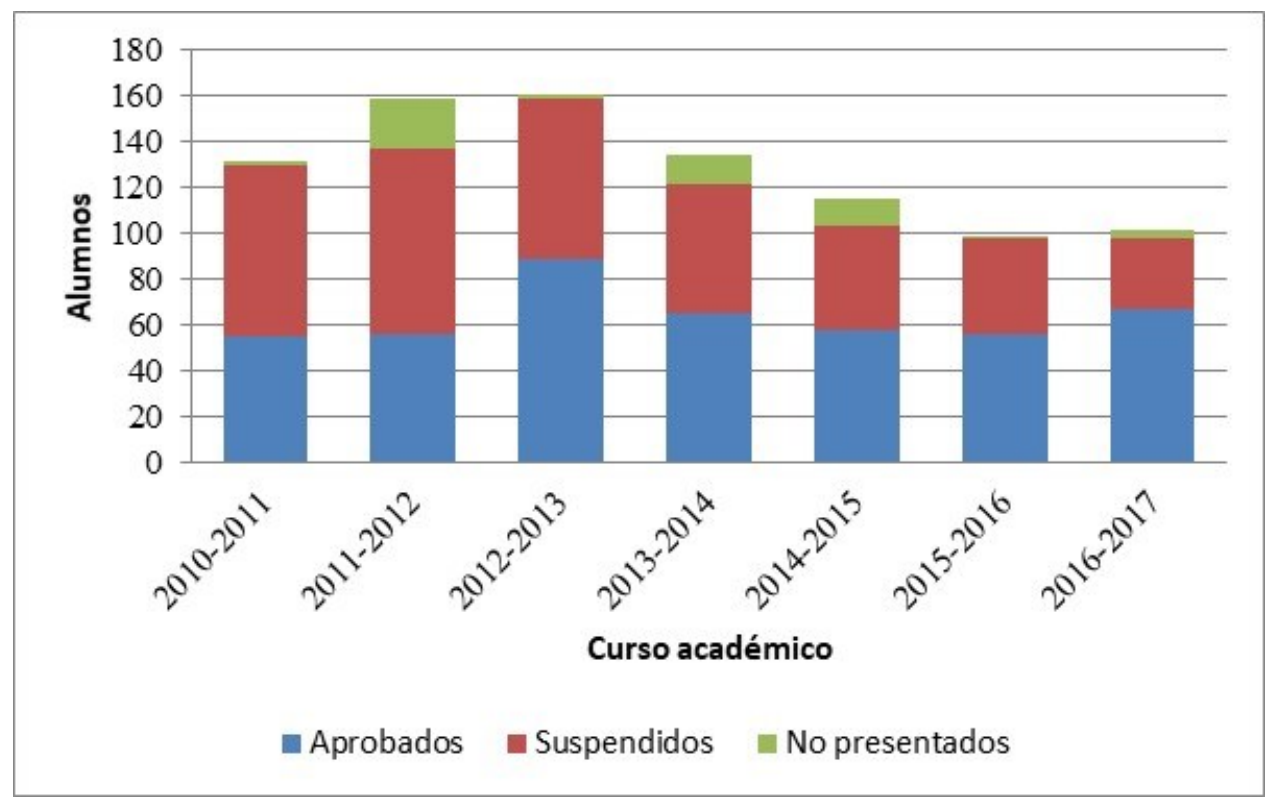

Figura 1. Estadísticas de la asignatura de EP. Fuente: Elaboración propia

\section{Metodología}

En la parte de microeconomía se diseñaron juegos en el aula mediante Kahoot! y en macroeconomía mediante Quizlet. Éstos se plantearon al finalizar los temas incluyendo los contenidos en los que los alumnos habían mostrado mayor dificultad, y se utilizaron unos 20 minutos para su realización. Los cuestionarios fueron planteados por los docentes de la asignatura con objeto de adaptarse lo más posible al nivel exigido, aunque en las dos plataformas muchos usuarios han dejado en abierto actividades preparadas. 
Para el análisis de las plataformas de juegos como herramienta para mejorar la motivación de los alumnos en el aula se realizará a primer lugar una descripción de las plataformas objeto de estudio, destacando las opciones que permiten su utilización como juegos en aula. Esto nos permitirá realizar una comparación entre ellas. La descripción del uso de la plataforma se realizará utilizando como ejemplo los cuestionarios realizados para la asignatura de Economía Política.

\subsection{Plataforma Kahoot!}

Kahoot! es una plataforma de aprendizaje gratuita basada en juegos. No requiere la instalación de ninguna aplicación informática, solamente conexión a internet. En la UPV existe wifi en todo su recinto, no supone coste alguno para los alumnos. Esta aplicación se engloba dentro del aprendizaje móvil electrónico (M-learning, en inglés) y de la ludificación (Gamification, en inglés). La idea es que el alumno aprenda jugando dentro del aula para que la experiencia de aprendizaje sea más motivadora.

La plataforma Kahoot! posibilita utilización de cuestionarios elaborados por el universo de docentes, así como la redacción de preguntas por parte del profesor de la propia asignatura, siendo esta última la práctica más habitual. En el primer caso, antes de su uso deberán ser revisados y corregidos para evitar errores o preguntas inapropiadas.

En la asignatura de EP se redactaron nuevos cuestionarios, adaptándose así a las necesidades específicas del aula. Para ello fue necesario registrarse en la plataforma (https://getKahoot.com/) y seleccionar la opción de realizar una nueva actividad, eligiendo la opción cuestionario (Quiz). En primer lugar, se introduce el título de la actividad que puede ir acompañado de una imagen. Seguidamente se van incluyendo las preguntas y sus correspondientes respuestas. Además, será necesario indicar el tiempo disponible para la lectura de cada pregunta y su respuesta múltiple, pudiendo establecer entre 5 y 120 segundos, y de 2 a 4 respuestas, permitiendo la opción de que haya más de una respuesta correcta (Figura 1).

La preparación del cuestionario permitió hacer visible la principal desventaja de esta plataforma: los caracteres están limitados tanto en el enunciado de la pregunta (95 caracteres) como en las posibles respuestas (60 caracteres). Esto dificulta el planteamiento de preguntas donde se trabaje más de un concepto, reduciendo su complejidad e impidiendo la relación entre distintas nociones básicas de la asignatura. Se trata, por tanto, de una técnica de aprendizaje de conocimientos aislados, no ofrece la visión de conjunto requerida en los exámenes de esta asignatura.

Una vez creado el cuestionario, para empezar el juego mediante el móvil, Tablet u ordenador el alumno deberá acceder a la Web (https://kahoot.it) a través de cualquier 
Mejorar la motivación en el aula mediante el uso de plataformas de aprendizaje basadas en juegos: Quizlet vs Kahoot

explorador de internet (safari, Chrome, Firefox, etc.) o una App. Existen 2 modalidades: en grupo o individual. En el caso de EP se seleccionó la segunda, todos los estudiantes tenían los dispositivos necesarios para su acceso. La web asigna un código PIN para su acceso, y tras introducirlo, los alumnos eligen su alias o nombre de usuario para identificarse. Posteriormente, se proyectan las preguntas y se debe seleccionar la respuesta que consideran correcta (Figura 2).

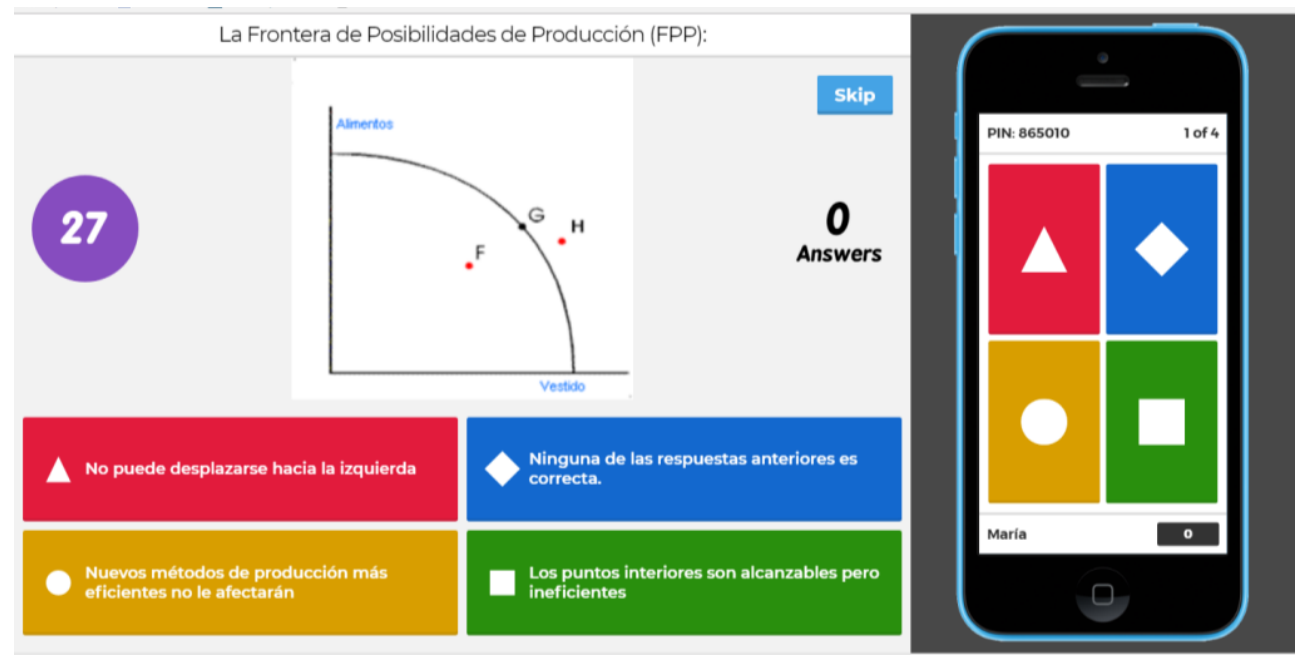

Figura 2. Ejemplo de una pregunta en Kahoot! de la parte de microeconomía (izquierda) y como lo visualiza el alumno en su terminal (derecha).Fuente: Elaboración propia mediante la utilización de la plataforma (https://getKahoot.com/)

Si se quiere utilizar el cuestionario en más de una ocasión, la plataforma permite aleatorizar las preguntas y respuestas, con el objeto que los alumnos no memoricen el orden de las éstas.

Al finalizar, se conoce la puntuación individual obtenida y se establece un ranking con las mismas. Ésta dependerá tanto de la cantidad de respuestas correctas, como de la velocidad de ejecución. El profesor pasa a un segundo plano, siendo los propios alumnos los protagonistas de la sesión educativa. Las funciones del docente se reducen a ser un mero presentador del juego, explicando su funcionamiento y respondiendo las posibles dudas que pudieran surgir. La aplicación permite exportar los resultados a Excel o incluirlos en Google Drive, para que el profesor pueda disponer de los mismos en el proceso de evaluación. Además se pueden consultar los aciertos y errores de cada alumno, así como el porcentaje de preguntas correctas e incorrectas y la nota media del ejercicio. 


\subsection{Plataforma Quizlet Live}

Al igual que la plataforma Kahoot!, el Quizlet también es gratuito, aunque dispone una versión de pago que incluye más opciones, tan solo se requiere disponer de conexión a internet. En esta plataforma el docente creará un grupo de trabajo o clase y elegirá una unidad de estudio para incluir las preguntas y la respuesta correcta de cada una. En la asignatura de EP se crearon cuestionarios para todos los temas de macroeconomía: contabilidad nacional, política fiscal y monetaria. En cada uno se ellos se incluían 20 conceptos de la materia con sus repuestas. Una vez elaborado el cuestionario, la plataforma permite la creación de diversas actividades, tanto de trabajo autónomo para el alumno como para juegos en el aula (Figura 3).

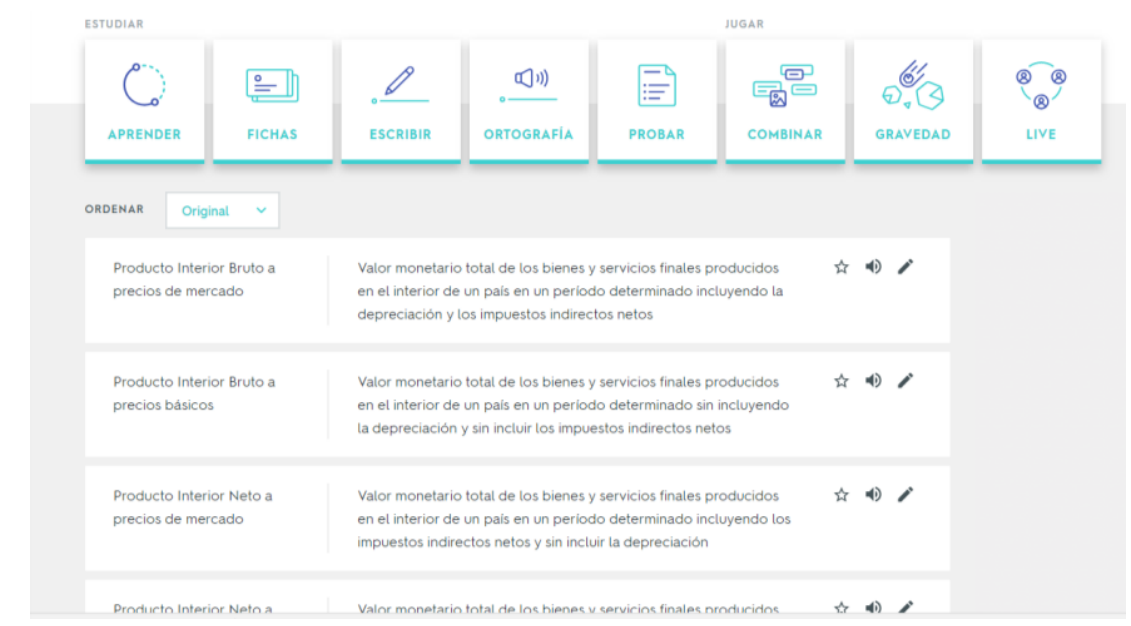

Figura 3. Opciones de actividades en Quizlet. Fuente: Elaboración propia del cuestionario en la web www.quizlet.live

La actividad de juego colaborativo en el aula es Quizlet Live. En esta actividad la web genera un código de acceso al cuestionario (www.quizlet.live). Esta versión combina competencia y colaboración para alentar a los estudiantes a aprender, formando equipos con los registrados (un mínimo de cuatro por grupo). Todos los alumnos de un mismo equipo reciben idéntica pregunta, pero cada compañero ve distintas respuestas posibles, y solo uno de ellos tiene la correcta, debiendo comunicarse para poder detectarla (Figura 4). Solo cuando la acierten podrán pasar a la siguiente pregunta. Si un equipo responde incorrectamente, vuelve al inicio del juego, por lo que responder rápidamente sin pensar no es la mejor opción. En cambio, cuando mejor sea el trabajo en grupo, más rápido avanzarán. El primer equipo en responder todas las preguntas correctamente ganará. 
Mejorar la motivación en el aula mediante el uso de plataformas de aprendizaje basadas en juegos: Quizlet vs Kahoot
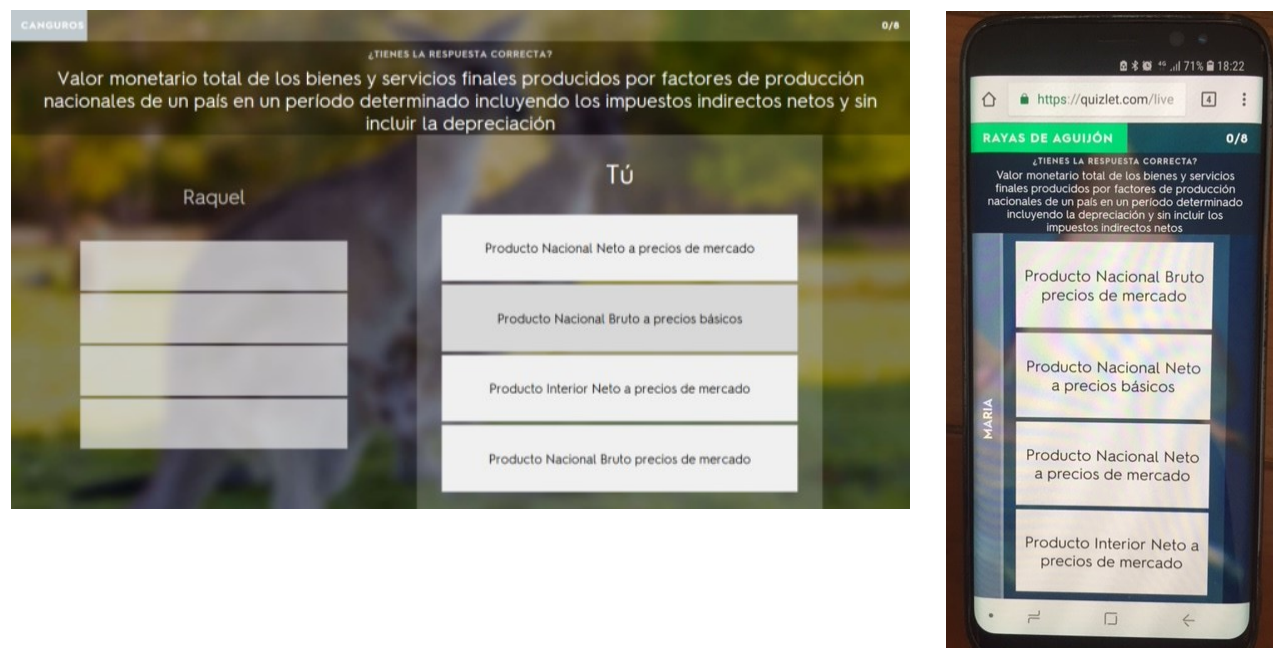

Figura 4. Ejemplo de cómo visualizan las preguntas cada alumno en una actividad de Quizlet live en el ordenador (izquierda) y en el móvil o Tablet (derecha). Fuente: Elaboración propia del cuestionario en la web www.quizlet.live

Mientras juegan, la pantalla del proyector funciona como un marcador interactivo, que sigue el avance de cada equipo y aviva la competencia (Figura 5). Cuando terminan el profesor realiza un repaso de los resultados de la clase. A diferencia de Kahoot!, éste no aporta información válida para la evaluación, dado que los equipos no avanzan hasta que hayan contestado correctamente. Además, el alcance es del equipo, y no individual como en el Kahoot!. Por tanto, aportan una retroalimentación del aprendizaje de los contenidos del conjunto del aula, pudiendo haber niveles de comprensión de los contenidos muy heterogéneos entre los alumnos.

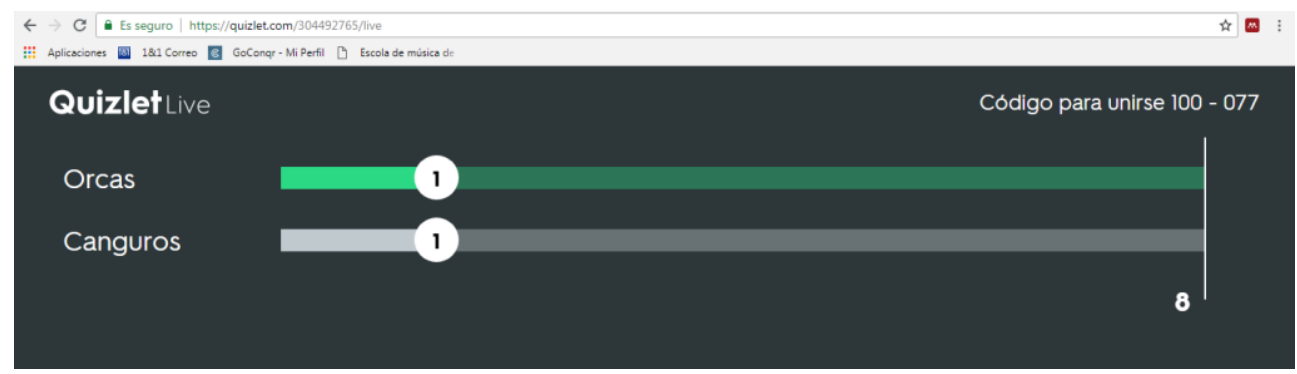

Figura 5: Ejemplo las puntuaciones de los equipos visualizadas en el proyector mientras dura la actividad de Quizlet live. Elaboración propia de los resultados de un cuestionario en la web www.quizlet.live 


\subsection{Aplicación a la asignatura de Economía Política}

Los contenidos de los temas de microeconomía se plantearon mediante 4 juegos con 15 preguntas tipo test, utilizando la plataforma Kahoot!. Dado que las respuestas se basaban en una descripción de los conceptos, y no se pretendía un grado de complejidad que implicara tener que relacionar conceptos, la posibles respuestas eran sencillas y se presentaban como afirmaciones muy cortas. Se programó para que contestestaran cada pregunta en menos de treinta segundos. Los contenidos del primer test trataban sobre conceptos básicos de economía y Frontera de Posibilidades de Producción, el segundo test sobre oferta y demanda, el tercero sobre elasticidades y el cuarto sobre maximización de beneficios en empresas en competencia perfecta. Se formaron grupos de entre 4 y 6 alumnos, dependiendo de los presentes en el aula el día del juego.

Los juegos con contenidos de los temas de macroeconomía se presentaron mediante Quizlet. Se plantearon 4 juegos en los que tenían que relacionar definiciones de conceptos o tipos de políticas con el nombre de la variable que los representa. En el primer juego se incluían variables de macroeconomía básica, en el segundo de contabilidad nacional, en el tercero de política fiscal y en el cuarto de política monetaria. Se formaron grupos, al igual que en la parte de microeconomía, de entre 4 y 6 alumnos.

\section{Resultados}

Los alumnos del grupo de tarde que asistieron a las clases presenciales contestaron a los cuestionarios, lo que supuso que como media participaron un $58 \%$ de los alumnos matriculados.

En los cuestionarios planteados mediante Kahoot! los resultados fueron los siguientes:

- El 100\% de los alumnos presentes en la sesión de teoría participó en el juego.

- El 93\% de las preguntas planteadas fueron contestadas por todos los grupos.

- El $43 \%$ de las respuestas contestadas fueron las correctas.

En los juegos planteados mediante Quizlet los resultados fueron los siguientes:

- El 100\% de los alumnos presentes en la sesión de teoría participó en el juego.

- El grupo ganador en cada juego contestó correctamente a todas las cuestiones utilizando dos o tres intentos para poder finalizar el juego.

- Cuando finalizaba el juego debido a que uno de los equipos había contestado correctamente a todas las preguntas, se comprobó en cada juego que el resto de grupos participantes habían contestado una media del $60-80 \%$ de preguntas de forma correcta. 
Mejorar la motivación en el aula mediante el uso de plataformas de aprendizaje basadas en juegos: Quizlet vs Kahoot

\section{Conclusiones}

Las dos plataformas permiten un ambiente más colaborativo de los alumnos en el aula, y por tanto una mejora en la atención y motivación hacia los contenidos de la asignatura. El acceso a ambas plataformas es gratuito y no presenta complejidad, siendo muy intuitivo. $\mathrm{Su}$ utilización está justificada por su gratuidad, diversión, utilidad y facilidad de manejo. Las actividades realizadas en ambas plataformas ofrecen distintos formatos de presentación (test, encuestas, etc.), lo que permite el juego en el aula y el aprendizaje autónomo de los alumnos fuera de las aulas, así como la autoevaluación. Los alumnos muestran satisfacción ante la utilización de estas herramientas e incitan al profesor a su uso más frecuente. Consideran que favorece la comunicación y la relación entre ellos, al generarse un ambiente más distendido dentro del aula, y la familiarización con las tecnologías de la información.

En el caso de la asignatura de EP los alumnos mostraron mayor implicación mediante la herramienta Quizlet live, dado que era menos conocida y les resultaba atractivo el trabajo en equipo. Por parte de los profesores se valora positivamente el incremento de la participación y motivación de los alumnos. Estas actividades permiten tener una retroalimentación activa y dinámica del proceso de aprendizaje individual y grupal, a través de las aportaciones de los alumnos durante el juego y la verificación de los resultados, e ir corrigiendo errores conceptuales que se han detectado a los alumnos.

En la plataforma Kahoot! Existen más desventajas durante la elaboración del cuestionario, dada la limitación de caracteres tanto en la pregunta como en las respuestas. En cambio, permite mayor grado de retroalimentación para el profesor, tanto del grado de comprensión de los contenidos de cada alumno como del proceso de aprendizaje individual de cada uno de ellos. Facilita la observación de los avances de cada alumno y, en algunos casos, una atención posterior más individualizada. En la plataforma Quizlet, en la versión de juego en el aula Quizlet live, se preguntas y respuestas pueden ser más largas pero la información recopilada por el docente para la retroalimentación del proceso de aprendizaje es más limitada.

Estas diferencias indican que, aunque se puedan trabajar competencias trasversales comunes en las dos plataformas, como son la comprensión e integración y la comunicación efectiva, además, en cada una de ellas se contemplan competencias transversales diferentes. Kahoot! potencia el aprendizaje permanente. En cambio Quizlet mejora el trabajo en equipo y liderazgo, y la planificación y gestión del tiempo. En la asignatura de EP impartida en el primer semestre del primer curso del GGAP se obtienen unos niveles dominio iniciales de estas competencias, pero en el caso de asignaturas en semestres más avanzados del grado se pueden conseguir mayores niveles de dominio, al ser los propios alumnos los que elaboren los cuestionarios y las actividades en estas plataformas. 


\section{Referencias}

Carreño, A. B., \& Vélez, S. C. (2016). Web 2.0 en educación superior: formación, actitud, uso, impacto, dificultades y herramientas. Digital Education Review, (28), 45-58.

Fuentes, M., Carrasco Andrino, M. D. M., Jiménez Pascual, A., Ramón Martín, A., Soler García, C., \& Vaello, T. (2016). El aprendizaje basado en juegos: experiencias docentes en la aplicación de la plataforma virtual" Kahoot".

Gutiérrez, A., Palacios, A., \& Torrego, L. (2010). Tribus digitales en las aulas universitarias. Comunicar, 34, 173-181.

Kay, R. H., \& LeSage, A. (2009). Examining the benefits and challenges of using audience response systems: A review of the literature. Computers \& Education, 53(3), 819-827.

Pintor, E., Gargantilla, P., Herreros, B., \& López del Hierro, M. (2014). Kahoot en docencia: una alternativa practica a los clickers.

Puertas, R., Martí, M. L., \& Calafat, C (2015). Análisis de las causas del bajo rendimiento de la asignatura de Economía Política. Conference on Innovation, Documentation and Teaching Technologies (INNODOCT 2015). Open Innovation and Coolhunting in Education (ISSN 978$84-9048-343-5) 255-261$

Calafat, C., Puertas, R., \&Martí, M. L. (2017). Mejora el flip teaching el resultado académico? Evidencia de la asignatura de Economía Política. Conference on Innovation, Documentation and Teaching Technologies (INNODOCT 2016). Lean education and innovation (ISSN 978-849048-528-6) $373-384$

Rodríguez, D. (2008). Nuevas tecnologías Web 2.0: Hacia una real democratización de la información y el conocimiento. Recuperado de: http://eprints. rclis. org/11814/1/RodriguezDianaTRABAJOelis. pdf.

Salgado, M. C., Gonzalez, M. J., \& Zamarra, M. D. L. M. (2014). Innovación y aplicación tecnológica en el ámbito de la Educación Superior universitaria. El empleo de los blogs en las universidades españolas. Historia y Comunicación Social, 18, 613-625.

Salinas, J. (2004). Innovación docente y uso de las TIC en la enseñanza universitaria. International Journal of Educational Technology in Higher Education (ETHE), 1(1).

Tabuenca, B., Verpoorten, D., Ternier, S., Westera, W., \& Specht, M. (2013). Fomento de la práctica reflexiva sobre el aprendizaje mediante el uso de tecnologías móviles. Revista de Educación a Distancia, (37).

Vivancos, J. (2009). La Competència digital i les TAC. Espiral.

Wang, A. I. (2015). The wear out effect of a game-based student response system. Computers \& Education, 82, 217-227. 